\title{
A New Approach for the Fractional Integral Operator in Time Scales with Variable Exponent Lebesgue Spaces
}

\author{
Lütfi Akın (iD)
}

check for updates

Citation: Akın, L. A New Approach for the Fractional Integral Operator in Time Scales with Variable Exponent Lebesgue Spaces. Fractal Fract. 2021, 5, 7. https://doi.org/10.3390/ fractalfract5010007

Received: 5 November 2020 Accepted: 5 January 2021 Published: 8 January 2021

Publisher's Note: MDPI stays neutral with regard to jurisdictional clai$\mathrm{ms}$ in published maps and institutional affiliations.

Copyright: () 2021 by the author. Licensee MDPI, Basel, Switzerland. This article is an open access article distributed under the terms and conditions of the Creative Commons Attribution (CC BY) license (https:// creativecommons.org/licenses/by/ $4.0 /)$.
Department of Business Administration, Mardin Artuklu University, 47200 Mardin, Turkey; lutfiakin@artuklu.edu.tr

\begin{abstract}
Integral equations and inequalities have an important place in time scales and harmonic analysis. The norm of integral operators is one of the important study topics in harmonic analysis. Using the norms in different variable exponent spaces, the boundedness or compactness of the integral operators are examined. However, the norm of integral operators on time scales has been a matter of curiosity to us. In this study, we prove the equivalence of the norm of the restricted centered fractional maximal diamond- $\alpha$ integral operator $\mathrm{M}_{\mathrm{a}, \delta}^{\mathrm{c}}$ to the norm of the centered fractional maximal diamond- $\alpha$ integral operator $M_{a}^{c}$ on time scales with variable exponent Lebesgue spaces. This study will lead to the study of problems such as the boundedness and compactness of integral operators on time scales.
\end{abstract}

Keywords: time scales; variable exponent; fractional integral; maximal operator

\section{Introduction}

The founder of the time scale theory is Stefan Hilger [1]. At the time, this theory caught the attention of many mathematicians who have demonstrated various aspects of integral inequalities, dynamic equations and integral operators on time scales [2-13]. For example, Li [4] demonstrated non-linear integral inequalities in two independent variables on time scales. Anastassiou [5] demonstrated some properties of fractional calculus on time scales. Uçar et al. [9] demonstrated fractional integral inequalities on time scales.

Dynamic equations and integral inequalities have many applications in different areas of science. Some areas are electrical engineering, fluid dynamics, quantum mechanics, physical problems, wave equations, heat transfer and economics [14-24]. Tisdell and Zaidi [15] demonstrated basic qualitative and quantitative results for solutions to non-linear dynamic equations on time scales with an application to economic modelling. Seadawy et al. [18] demonstrated non-linear wave solutions of the Kudryashov-Sinelshchikov dynamical equation in mixtures of liquid-gas bubbles under the consideration of heat transfer and viscosity. Akin [25] demonstrated fractional integral type inequalities on time scales. Higgins [26] demonstrated asymptotic behavior of second-order nonlinear dynamic equations on time scales. Ozturk and Higgins [27] demonstrated limit behaviors of non-oscillatory solutions of three-dimensional time scale systems.

The variable exponent, Lebesgue space $L^{p(.)}$, is one of the cornerstones of harmonic analysis. Mathematicians working in this field have comprehensively analyzed the operators and inequalities in the variable exponent, Lebesgue space $\mathrm{L}^{\mathrm{p}(.)}$ [28-37]. Akin and Dusunceli [38] demonstrated a new approach for weighted Hardy's operator in "variable exponent Lebesgue spaces" (VELS). This work also has been stimulated by problems of elasticity, fluid dynamics, electrorheological liquids and calculus of variations.

In variable exponential spaces, problems such as the boundedness and compactness of integral operators, take an important place. The concept of norms has the most important place in solving these problems. If we can obtain the norms of integral operators by the method we apply, then we can see the boundedness and compactness of these 
operators. However, there are almost no studies on the problems of time scales. Our main purpose in this study is to examine the equivalence of the norms of fractional integral operators. Thanks to these results, we will be able to establish the constraint and compactness conditions of integral operators on time scales.

The organization of this article is as follows. In Section 2, we give necessary definitions, lemmas and theorems. In Section 3, we prove the equivalence of the norm variable exponent $\mathrm{L}^{\mathrm{p}(.)}$ of the restricted centered fractional maximal diamond- $\alpha$ integral $\mathrm{M}_{\mathrm{a}, \delta}^{\mathrm{c}} \mathrm{b}$ to the norm variable exponent $\mathrm{L}^{\mathrm{p}(\cdot)}$ of the centered fractional maximal diamond- $\alpha$ integral $\mathrm{M}_{\mathrm{a}}^{\mathrm{c}}$ for all $0<\delta<\infty$ and $1 \leq \mathrm{p}(\mathrm{x})<\infty$ on time scales. In Section 4 , we give the conclusion.

\section{Materials and Methods}

In this section, we provide necessary concepts and statements related to time scale and variable exponent Lebesgue space. The reader can refer to the monographs [1-43] for details.

Definition 1. [39] Let $\Omega \subset \mathbb{R}^{n}$ be an open set. The fractional maximal operator $M_{a} f$ is defined as follows

$$
M_{a} f(t)=\sup _{B \ni t} \frac{1}{|B|^{1-\frac{a}{n}}} \int_{B \cap \Omega}|f(y)| d y,
$$

for $0<a<n$. In the limiting case $a=0$, the fractional maximal operator reduces to the Hardy-Littlewood maximal operator.

Let $L^{p(\cdot)}(\Omega)$ denote the space of the measurable and integrable functions on $\Omega$, such that for $\lambda>0$, with norm

$$
\|f\|_{p(.), \Omega}=\inf \left\{\lambda>0: \int_{\Omega}\left(\frac{|f(t)|}{\lambda}\right)^{p(t)} d t \leq 1\right\},
$$

where $p():. \Omega \rightarrow[1, \infty)$ is a measurable function (for details, see $[25,40]$ ). These spaces are also the variable exponent Lebesgue spaces $L^{p(.)}$.

Now let us define some properties of the centered fractional maximal operator which will help us prove our results. We know that the centered fractional maximal operator is defined by

$$
M_{a}^{c} f(t)=\sup _{r>0} \frac{1}{|B(t, r)|^{1-\frac{a}{n}}} \int_{B(t, r) \cap \Omega}|f(\tau)| d \tau,
$$

and the uncentered fractional maximal operator is defined by

$$
M_{a} f(t)=\sup _{B \ni t} \frac{1}{|B|^{1-\frac{a}{n}}} \int_{B \cap \Omega}|f(\tau)| d \tau
$$

for $0<a<n$ and $t \in \mathbb{R}^{n}$, where the supremum is again taken over all balls $B$, which contain $t$ (for details, see $[25,29,37])$. We well know that the fractional maximal operator plays an important role in harmonic analysis. The restricted operators have important properties (for details, see $[28,41]$ ).

$$
M_{a}^{c} f(t) \leq M_{a} f(t) \leq 2^{m} M_{a}^{c} f(t), \quad \forall t \in \mathbb{R}^{m} .
$$

Let us define the restricted centered fractional maximal operator and the restricted uncentered fractional maximal operator, respectively,

$$
M_{a, \delta}^{c} f(t)=\sup _{\delta>r>0} \frac{1}{|B(t, r)|^{1-\frac{a}{n}}} \int_{B(t, r) \cap \Omega}|f(\tau)| d \tau,
$$


and

$$
M_{a, \delta} f(t)=\sup _{\delta>r>0,|x-t|<r} \frac{1}{|B|^{1-\frac{a}{n}}} \int_{B(x, r) \cap \Omega}|f(\tau)| d \tau,
$$

for $t \in \mathbb{R}^{m}, 0<a<n$ and $\delta \in \mathbb{R}_{+}$(for details, see [28,29,41]). It is clear that using (5) and (6), we can write the following inequalities

$$
M_{a, \delta}^{c} f(t) \leq M_{a, \theta}^{c} f(t) \leq M_{a}^{c} f(t)
$$

and

$$
M_{a, \delta} f(t) \leq M_{a, \theta} f(t) \leq M_{a} f(t),
$$

for $\delta \leq \theta, \forall t \in \mathbb{R}^{m}$. As a result, again if we use (5)-(6) and $\delta \leq \theta$, then we can write the following inequalities and

$$
\begin{gathered}
\left\|M_{a, \delta} f(t)\right\|_{L^{p(.)}\left(\mathbb{R}^{m}\right) \rightarrow L^{p(.)}\left(\mathbb{R}^{m}\right)} \leq\left\|M_{a, \theta} f(t)\right\|_{L^{p(.)}\left(\mathbb{R}^{m}\right) \rightarrow L^{p(\cdot)}\left(\mathbb{R}^{m}\right)} \\
\leq\left\|M_{a} f(t)\right\|_{L^{p(.)}\left(\mathbb{R}^{m}\right) \rightarrow L^{p(.)}\left(\mathbb{R}^{m}\right)}
\end{gathered}
$$

for $1<p(.) \leq \infty, t \in \mathbb{R}^{m}$ and some real positive numbers $\delta, \theta$. We have seen here that the norm of the restricted operator is less than the norm of the unrestricted operator. The normed inequalities obtained above will help us prove our results.

To prove our main results, we first provide some definitions and lemmas which will be used as follows.

Definition 2. [29] If $f$ is a measurable function on $\mathbb{R}^{m}$, then distribution function $d_{f}$ on $[0,+\infty]$ is defined by

$$
d_{f}(\beta)=\left|\left\{x \in \mathbb{R}^{m}:|f(x)|>\beta\right\}\right|,
$$

where $\left|\left\{x \in \mathbb{R}^{m}:|f(x)|>\beta\right\}\right|$ is the Lebesgue measurable of the measurable of $\left\{x \in \mathbb{R}^{m}:|f(x)|>\beta\right\}$.

Lemma 1. [30] If $f \in L^{p}\left(\mathbb{R}^{m}\right)$ with $0<p<\infty$, then we have

$$
\|f\|_{L^{p}\left(\mathbb{R}^{m}\right)}^{p}=p \int_{0}^{\infty} \beta^{p-1} d_{f}(\beta) d \beta .
$$

Lemma 2. [31] Let $\pi$ be a positive measure on $\mathcal{M}$ such that $\mathcal{M}$ is a $\sigma$-algebra. If $B_{1} \subset B_{2} \subset$ $B_{3}, \ldots, B_{m} \in \mathcal{M}$, and $B=\cup_{m=1}^{\infty} B_{m}$, then $\lim _{m \rightarrow \infty} \pi\left(B_{m}\right)=\pi(B)$.

Using Lemma 2, we can formulate the following conclusions.

Lemma 3. (Lemma 2.3, [29]) If operators $M_{a}^{c}$ and $M_{a, \delta}^{c}$ are defined as in (2) and (5), then the equality

$$
d_{M_{a}^{c} f}(\beta)=\lim _{\delta \rightarrow \infty} d_{M_{a, \delta}^{c} f}(\beta),
$$

holds $\forall f \in L^{p}\left(\mathbb{R}^{m}\right)$ and $\beta>0$.

Lemma 4. (Lemma 2.4, [29]) Ifoperators $M_{a}$ and $M_{a, \delta}$ are defined as in (3) and (6), then the equality

$$
d_{M_{a} f}(\beta)=\lim _{\delta \rightarrow \infty} d_{M_{a, \delta} f}(\beta),
$$

holds $\forall f \in L^{p}\left(\mathbb{R}^{m}\right)$ and $\beta>0$. 
Lemma 5. [29] For $p>1$ and $0<\epsilon$, there exists a function $h \in C_{-} c^{\infty}\left(R_{m} m\right)$ such that

$$
\begin{aligned}
& \qquad\left\|M_{a}^{c}\right\|_{L^{p}\left(\mathbb{R}^{m}\right) \rightarrow L^{p}\left(\mathbb{R}^{m}\right)}-\epsilon \leq \frac{\left\|M_{a}^{c} h\right\|_{L^{p}\left(\mathbb{R}^{m}\right)}}{\|h\|_{L^{p}\left(\mathbb{R}^{m}\right)}}, \\
& \text { where }\left\|M_{a}^{c}\right\|_{L^{p}\left(\mathbb{R}^{m}\right) \rightarrow L^{p}\left(\mathbb{R}^{m}\right)}=\sup _{\|h\|_{L^{p}\left(\mathbb{R}^{m}\right)} \neq 0} \frac{\left\|M_{a}^{c} h\right\|_{L^{p}\left(\mathbb{R}^{m}\right)}}{\|h\|_{L^{p}\left(\mathbb{R}^{m}\right)}} .
\end{aligned}
$$

Let us give information about the time scales that will help us in our work.

A time scale $\mathbb{T}$ is a nonempty closed subset of $\mathbb{R}$ (for details, see $[19,20])$. Let $[a, b]$ be an arbitrary closed interval on time scale $\mathbb{T}$. The time scale interval $[a, b]_{\mathbb{T}}$ is denoted by $[a, b] \cap \mathbb{T}$ (see p. $7[42])$.

Definition 3. [19] The mappings $\sigma, \rho: \mathbb{T} \rightarrow \mathbb{T}$ are defined by $\sigma(t)=\inf \{s \in T: s>t\}$, $\rho(t)=\sup \{s \in \mathbb{T}: s>t\}$ for $t \in \mathbb{T}$. Respectively, $\sigma(t)$ is forward jump operator and $\rho(t)$ is backward jump operator.

If $\sigma(t)>t$, then $t$ is right-scattered and if $\sigma(t)=t$, then $t$ is called right-dense. If $\rho(t)<t$, then $t$ is left-scattered and if $\rho(t)=t$, then $t$ is called left-dense.

Definition 4. [19] Let two mappings $\mu, \vartheta: \mathbb{T} \rightarrow \mathbb{R}^{+}$, such that $\mu(t)=\sigma(t)-t, \vartheta(t)=t-\rho(t)$. Here, the mappings $\mu(t)$ and $\vartheta(t)$ are called graininess mappings.

If $\mathbb{T}$ has a left-scattered maximum $m$, then $\mathbb{T}^{k}=\mathbb{T}-\{m\}$. Otherwise $\mathbb{T}^{k}=\mathbb{T}$.

$\mathbb{T}^{k}$ is defined as follows (for details, see $[19,20,42]$ )

$$
\mathbb{T}^{k}=\left\{\begin{array}{c}
\mathbb{T} \backslash(\rho \sup \mathbb{T}, \sup \mathbb{T}], \quad \text { if } \sup \mathbb{T}<\infty, \\
\mathbb{T}, \quad \text { if } \quad \sup \mathbb{T}=\infty,
\end{array}\right.
$$

by the same way

$$
\mathbb{T}_{k}=\left\{\begin{array}{cr}
\mathbb{T} \backslash[\inf \mathbb{T}, \sigma(\inf \mathbb{T})], & |\inf \mathbb{T}|<\infty, \\
\mathbb{T}, & \inf \mathbb{T}=-\infty
\end{array}\right.
$$

Assume that $h: \mathbb{T} \rightarrow \mathbb{R}$ is a function.

(i) If $h$ is $\Delta$-differentiable at point $t\left(t \in \mathbb{T}^{k}(t \neq \min \mathbb{T})\right)$, then $h$ is continuous at point $t$.

(ii) If $h$ is left continuous at point $t$ and $t$ is right-scattered, then $h$ is $\Delta$-differentiable at point $t$,

$$
h^{\Delta}(t)=\frac{h^{\sigma}(t)-h(t)}{\mu(t)}
$$

Let $t$ be right-dense.

(iii) If $h$ is $\Delta$-differentiable at point $t$ and $\lim _{s \rightarrow t} \frac{h(t)-h(s)}{t-s}$, then

$$
h^{\Delta}(t)=\lim _{s \rightarrow t} \frac{h(t)-h(s)}{t-s} .
$$

(iv) If $h$ is $\Delta$-differentiable at point $t$, then $h^{\sigma}(t)=h(t)+\mu(t) h^{\Delta}(t)$.

Remark 1. (For details, see page 3 in [42]) If $\mathbb{T}=\mathbb{R}$, then $h^{\Delta}(t)=h^{\prime}(t)$, and if $\mathbb{T}=\mathbb{Z}$, then $h^{\Delta}(t)$ reduces to $\Delta h(t)$. 
Definition 5. [20] If $H: \mathbb{T} \rightarrow \mathbb{R}$ is defined as $\Delta$-antiderivative of $h: \mathbb{T} \rightarrow \mathbb{R}$, then $H^{\Delta}=h(t)$ holds for $\forall t \in \mathbb{T}$ and we define the $\Delta$-integral of $h$ by

$$
\int_{s}^{t} h(\tau) \Delta \tau=H(t)-H(s)
$$

for $s, t \in \mathbb{T}$.

We now give similar definitions for the nabla operator.

Definition 6. [20] Where $h: \mathbb{T}_{k} \rightarrow \mathbb{R}$ is called $\nabla$-differentiable at $t \in \mathbb{T}_{k}$. If $\varepsilon>0$, then there exists a neighborhood $V$ of $t$ such that

$$
\left|h(\rho(t))-h(s)-h^{\nabla}(t)(\rho(t)-s)\right| \leq \varepsilon|\rho(t)-s|,
$$

for $\forall s \in V$.

Definition 7. [20] Where $H: \mathbb{T} \rightarrow \mathbb{R}$ is called a $\nabla$-antiderivative of $h: \mathbb{T} \rightarrow \mathbb{R}$, then we define

$$
\int_{s}^{t} h(\tau) \nabla \tau=H(t)-H(s),
$$

for $s, t \in \mathbb{T}$. Let $f(t)$ be differentiable on $\mathbb{T}$ for $\alpha, t \in \mathbb{T}$. Then, we define $f^{\diamond_{\alpha}}(t)$ by

$$
f^{\diamond_{\alpha}}(t)=\alpha f^{\Delta}(t)+(1-\alpha) f^{\nabla}(t)
$$

for $0 \leq \alpha \leq 1$.

Proposition 1. [20] If we have $f, h: \mathbb{T} \rightarrow \mathbb{R}, \diamond_{\alpha}$-differentiable for $\alpha, t \in \mathbb{T}$, then

(i) $\quad f+h: \mathbb{T} \rightarrow \mathbb{R}$ is $\diamond_{\alpha}$-differentiable for $t \in \mathbb{T}$ with $(f+h)^{\diamond_{\alpha}}(t)=f^{\diamond_{\alpha}}(t)+h^{\diamond_{\alpha}}(t)$.

(ii) Let $k \in \mathbb{R}, k f: \mathbb{T} \rightarrow \mathbb{R}$ is $\diamond_{\alpha}$-differentiable for $\alpha, t \in \mathbb{T}$ with $(k f)^{\diamond_{\alpha}}(t)=k f^{\diamond_{\alpha}}(t)$.

(iii) $f, h: \mathbb{T} \rightarrow \mathbb{R}$ is $\diamond_{\alpha}$-differentiable for $\alpha, t \in \mathbb{T}$ with

$$
(f h)^{\diamond_{\alpha}}(t)=f^{\diamond_{\alpha}}(t) h(t)+\alpha f^{\sigma}(t) h^{\Delta}(t)+(1-\alpha) f^{\rho}(t) h^{\nabla}(t) .
$$

Definition 8. [20] If $f: \mathbb{T} \rightarrow \mathbb{R}$ is integrable and $\alpha, b, t \in \mathbb{T}$, then

$$
\int_{b}^{t} f(\delta) \diamond_{\alpha} \delta=\alpha \int_{b}^{t} f(\delta) \Delta \delta+(1-\alpha) \int_{b}^{t} f(\delta) \nabla \delta
$$

for $0 \leq \alpha \leq 1$.

Definition 9. [12,25] If $f \in C_{r d}(\mathbb{T}, \mathbb{R})$ and $t \in \mathbb{T}^{k}$, then

$$
\int_{t}^{\sigma(t)} f(\tau) \diamond_{\alpha} \tau=\mu(t) f(t) .
$$

Here, we can define the fractional maximal diamond- $\alpha$ integral

$$
M_{a} f(t)=\sup _{B \ni t} \frac{1}{|B|^{\frac{m-a}{m}}} \int_{a}^{t} f(x) \diamond_{\alpha} x
$$


where $f \in L_{1}([a, t] \cap \mathbb{T})$ and $M_{a} f \in L([a, t] \cap \mathbb{T})$.

Now, we can define the restricted centered fractional maximal diamond- $\alpha$ integral operator and the restricted uncentered fractional maximal diamond- $\alpha$ integral operator,

$$
M_{a, \delta}^{c} f(t)=\sup _{\delta>r>0} \frac{1}{|B(t, r)|^{1-\frac{a}{m}}} \int_{B(t, r) \cap \Omega}|f(\tau)| \diamond_{\alpha} \tau
$$

and

$$
M_{a, \delta} f(t)=\sup _{\delta>r>0,|x-t|<r} \frac{1}{|B|^{1-\frac{a}{m}}} \int_{B(x, r) \cap \Omega}|f(\tau)| \diamond_{\alpha} \tau,
$$

for $t \in \mathbb{R}^{m}$ and $\delta \in \mathbb{R}_{+}$(for details, see [28]).

Definition 10. [35] Let $p: \Phi \rightarrow[1, \infty)$ be a measurable function for $\Phi \subset \mathbb{R}^{m}$ and $L^{p(.)}$ be the space of all measurable functions on open set $\Phi$, such that

$$
\int_{\Phi}\left(\frac{|f(t)|}{\lambda}\right)^{p(t)} d t \leq \infty,
$$

for some $\lambda>0$. The norm in $L^{p(x)}$ is the generalization of the norm in $L^{p}$ ( $p$ is constant). The Luxemburg norm in $L^{p(x)}$ is defined as follows:

$$
\|f\|_{L^{p(.)}}=\inf \left\{\lambda>0: \int_{\Phi}\left(\frac{|f(t)|}{\lambda}\right)^{p(t)} d t \leq 1\right\} .
$$

Theorem 1. (See [Theorem 2, in [21]) If $h$ is $\Delta$-integrable on $[a, b]$, then $|h|$ is $\Delta$-integrable on $[a, b]$ and we have

$$
\left|\int_{a}^{b} h(\tau) \Delta \tau\right| \leq \int_{a}^{b}|h(\tau)| \Delta \tau
$$

Theorem 2. [40] Let $M_{a, \delta} f$ be defined by (6), and let $\delta>0$. Then

$$
\left\|M_{a, \delta} f\right\|_{L^{p(x)}\left(\mathbb{R}^{m}\right) \rightarrow L^{p(x)}\left(\mathbb{R}^{m}\right)}=\left\|M_{a} f\right\|_{L^{p(x)}\left(\mathbb{R}^{m}\right) \rightarrow L^{p(x)}\left(\mathbb{R}^{m}\right)}
$$

holds for $1 \leq p(x)<\infty$.

Proof. From the definition of the operator $M_{a, \delta} f$ in (6), we obtain

$$
\begin{gathered}
M_{a, \delta} f(\delta x)=\sup _{\delta>r>0,|t-\delta x|<r} \frac{1}{|B|^{1-\frac{a}{m}}} \int_{B(t, r)}|f(y)| \Delta y \\
=\sup _{\delta>r>0,|t-x|<\frac{r}{\delta}} \frac{1}{v_{m} r^{1-\frac{a}{m}}} \int_{|t|<r}|f(\delta t-y)| \Delta y \\
=\sup _{\delta>r>0,|t-x|<\frac{r}{\delta}} \frac{\delta^{1-\frac{a}{m}}}{v_{m} r^{1-\frac{a}{m}}} \int_{|t|<\frac{r}{\delta}}|f(\delta(t-y))| \Delta y \\
=\sup _{1>\frac{r}{\delta}>0,|t-x|<\frac{r}{\delta}} \frac{1}{v_{m}\left(\frac{r}{\delta}\right)^{1-\frac{a}{m}}} \int_{|t|<\frac{r}{\delta}}\left|\left(\tau_{\delta} f\right)(t-y)\right| \Delta y \\
=\sup _{1>r>0,|t-x|<r} \frac{1}{v_{m} r^{1-\frac{a}{m}}} \int_{|t|<r}\left|\left(\tau_{\delta} f\right)(x-y)\right| \Delta y \\
=M_{a, 1}\left(\tau_{\delta} f\right)(x),
\end{gathered}
$$


where $v_{m}$ is the volume of the unit ball in $R^{m}$ and the dilation operator $\tau_{\delta}$ is defined as follows:

$$
\left(\tau_{\delta} f\right)(x)=f(\delta x)
$$

for $\delta>0$ and $x \in R^{m}$. It follows from (12) that

$$
\frac{\left\|M_{a, \delta} f\right\|_{L^{p(x)}\left(\mathbb{R}^{m}\right)}}{\|f\|_{L^{p(x)}\left(\mathbb{R}^{m}\right)}}=\frac{\left\|M_{a, \delta} f(\delta)\right\|_{L^{p(x)}\left(\mathbb{R}^{m}\right)}}{\|f(\delta)\|_{L^{p(x)}\left(\mathbb{R}^{m}\right)}}=\frac{\left\|M_{a, 1}\left(\tau_{\delta} f\right)\right\|_{L^{p(x)}\left(\mathbb{R}^{m}\right)}}{\left\|\tau_{\delta} f\right\|_{L^{p(x)}\left(\mathbb{R}^{m}\right)}} .
$$

Taking the supremum over all $f \in L^{p(x)}\left(\mathbb{R}^{m}\right)$ with $\|f\|_{L^{p(x)}\left(\mathbb{R}^{m}\right)} \neq 0$ for the two sides of the above equation, we have

$$
\left\|M_{a, \delta} f\right\|_{L^{p(x)}\left(\mathbb{R}^{m}\right) \rightarrow L^{p(x)}\left(\mathbb{R}^{m}\right)}=\left\|M_{a, 1} f\right\|_{L^{p(x)}\left(\mathbb{R}^{m}\right) \rightarrow L^{p(x)}\left(\mathbb{R}^{m}\right)^{\prime}}
$$

for all $\delta>0$ and $1 \leq p(x)<\infty$.

Next, we will prove that

$$
\left\|M_{a, \delta} f\right\|_{L^{p(x)}\left(\mathbb{R}^{m}\right) \rightarrow L^{p(x)}\left(\mathbb{R}^{m}\right)}=\left\|M_{a} f\right\|_{L^{p(x)}\left(\mathbb{R}^{m}\right) \rightarrow L^{p(x)}\left(\mathbb{R}^{m}\right)} .
$$

We will use Equation (13) for proof. If $f \in L^{p(x)}\left(\mathbb{R}^{m}\right)$, then we have $M f \in L^{p(x)}\left(\mathbb{R}^{m}\right)$. From Lemma 1, Lemma 4 and Equation (13), we obtain

$$
\begin{gathered}
\left\|M_{a}\right\|_{L^{p(x)}}^{p(x)}\left(\mathbb{R}^{m}\right) \rightarrow L^{p(x)}\left(\mathbb{R}^{m}\right) \\
=\int_{0}^{\infty} \mu^{p(x)-1} \lim _{\delta \rightarrow \infty} \mu_{M_{a, \delta} f}(\mu) \Delta \mu \\
=\lim _{\delta \rightarrow \infty} \int_{0}^{\infty} \mu^{p(x)-1} d_{M_{a, \delta} f}(\mu) \Delta \mu \\
=\lim _{\delta \rightarrow \infty}\left\|M_{a, \delta}\right\|_{L^{p(x)}\left(\mathbb{R}^{m}\right)}^{p(x)} \\
\leq \lim _{\delta \rightarrow \infty}\left\|M_{a, \delta}\right\|_{L^{p(x)}\left(\mathbb{R}^{m}\right) \rightarrow L^{p(x)}\left(\mathbb{R}^{m}\right)}^{p(x)}\|f\|_{L^{p(x)}\left(\mathbb{R}^{m}\right)}^{p(x)} \\
=\left\|M_{a, 1}\right\|_{L^{p(x)}\left(\mathbb{R}^{m}\right) \rightarrow L^{p(x)}\left(\mathbb{R}^{m}\right)}^{p(x)}\|f\|_{L^{p(x)}\left(\mathbb{R}^{m}\right)}^{p(x)} .
\end{gathered}
$$

Now, taking advantage of inequality (14), we get the following inequality

$$
\left\|M_{a}\right\|_{L^{p(x)}\left(\mathbb{R}^{m}\right) \rightarrow L^{p(x)}\left(\mathbb{R}^{m}\right)}^{p(x)} \geq\left\|M_{a, 1}\right\|_{L^{p(x)}\left(\mathbb{R}^{m}\right) \rightarrow L^{p(x)}\left(\mathbb{R}^{m}\right)}^{p(x)} .
$$

Hence, we obtain from (14) that

$$
\left\|M_{a}\right\|_{L^{p(x)}\left(\mathbb{R}^{m}\right) \rightarrow L^{p(x)}\left(\mathbb{R}^{m}\right)}^{p(x)}=\left\|M_{a, 1}\right\|_{L^{p(x)}\left(\mathbb{R}^{m}\right) \rightarrow L^{p(x)}\left(\mathbb{R}^{m}\right)}^{p(x)} .
$$

\section{Main Results}

In this section we give statements and proofs of our results.

Theorem 3. Suppose that $M_{a, \delta}^{c} f(x)$ is defined by (5). Then

$$
\left\|M_{a, \delta}^{c}\right\|_{L^{p(x)}\left(\mathbb{R}^{m}\right) \rightarrow L^{p(x)}\left(\mathbb{R}^{m}\right)}=\left\|M_{a}^{c}\right\|_{L^{p(x)}\left(\mathbb{R}^{m}\right) \rightarrow L^{p(x)}\left(\mathbb{R}^{m}\right)}
$$

holds for $\delta>0$ and $1 \leq p(x)<\infty$. 
Proof. For $0<\delta<\infty$, we first prove

$$
\begin{aligned}
& \left\|M_{a, \delta}^{c}\right\|_{L^{p(x)}\left(\mathbb{R}^{m}\right) \rightarrow L^{p(x)}\left(\mathbb{R}^{m}\right)}=\left\|M_{a, 1}^{c}\right\|_{L^{p(x)}\left(\mathbb{R}^{m}\right) \rightarrow L^{p(x)}\left(\mathbb{R}^{m}\right)} . \\
& M_{a, \delta}^{c} f(x)=\sup _{\delta>r>0} \frac{1}{|B(x, r)|^{1-\frac{a}{m}}} \int_{B(x, r) \cap \Omega}|f(y)| \diamond_{\alpha} y=\sup _{\delta>r>0} \frac{1}{v_{m} r^{1-\frac{a}{m}}} \int_{|y| \leq r}|f(x-y)| \diamond_{\alpha} y, \\
& M_{a, \delta}^{c} f(\delta x)=\sup _{\delta>r>0} \frac{1}{v_{m} r^{1-\frac{a}{m}}} \int_{|y| \leq r}|f(\delta x-y)| \diamond_{\alpha} y \\
& =\sup _{\delta>r>0} \frac{\delta^{m}}{v_{m} r^{1-\frac{a}{m}}} \int_{|y| \leq \frac{r}{\delta}}|f(\delta x-\delta y)| \diamond_{\alpha} y \\
& =\sup _{1>\frac{r}{\delta}>0} \frac{1}{v_{m}\left(\frac{r}{\delta}\right)^{1-\frac{a}{m}}} \int_{|y| \leq \frac{r}{\delta}}|f(\delta x-\delta y)| \diamond_{\alpha} y \\
& =\sup _{1>r>0} \frac{1}{v_{m} r^{1-\frac{a}{m}}} \int_{|y| \leq r}|f(\delta x-\delta y)| \diamond_{\alpha} y \\
& =M_{a, 1}^{c} f(\delta x),
\end{aligned}
$$

for $0<\delta<\infty$ and $x \in \mathbb{R}^{m}$, where $v_{m}$ is the volume of the unit ball in $\mathbb{R}^{m}$. Hence,

for $0<\delta<\infty$ and $x \in \mathbb{R}^{m}$. If we use (17), then we have

$$
\frac{\left\|M_{a, \delta}^{c}\right\|_{L^{p(x)}\left(\mathbb{R}^{m}\right)}}{\|f\|_{L^{p(x)}\left(\mathbb{R}^{m}\right)}}=\frac{\left\|M_{a, \delta}^{c} f(\delta x)\right\|_{L^{p(x)}\left(\mathbb{R}^{m}\right)}}{\|f(\delta x)\|_{L^{p(x)}\left(\mathbb{R}^{m}\right)}}=\frac{\left\|M_{a, 1}^{c} f(\delta x)\right\|_{L^{p(x)}\left(\mathbb{R}^{m}\right)}}{\|f(\delta x)\|_{L^{p(x)}\left(\mathbb{R}^{m}\right)}} .
$$

If supremum is taken over all the $f \in L^{p(x)}\left(\mathbb{R}^{m}\right)$ for the two sides of (18), we have

$$
\left\|M_{a, \delta}^{c}\right\|_{L^{p(x)}\left(\mathbb{R}^{m}\right) \rightarrow L^{p(x)}\left(\mathbb{R}^{m}\right)}=\left\|M_{a, 1}^{c}\right\|_{L^{p(x)}\left(\mathbb{R}^{m}\right) \rightarrow L^{p(x)}\left(\mathbb{R}^{m}\right)} .
$$

Next, we will use Equation (19) to prove

$$
\left\|M_{a, \delta}^{c}\right\|_{L^{p(x)}\left(\mathbb{R}^{m}\right) \rightarrow L^{p(x)}\left(\mathbb{R}^{m}\right)}=\left\|M_{a}^{c}\right\|_{L^{p(x)}\left(\mathbb{R}^{m}\right) \rightarrow L^{p(x)}\left(\mathbb{R}^{m}\right)^{\prime}}
$$

for $\forall \delta>0$ and $1 \leq p(x)<\infty$. We just need to prove

$$
\left\|M_{a, \delta}^{c}\right\|_{L^{p(x)}\left(\mathbb{R}^{m}\right) \rightarrow L^{p(x)}\left(\mathbb{R}^{m}\right)} \geq\left\|M_{a}^{c}\right\|_{L^{p(x)}\left(\mathbb{R}^{m}\right) \rightarrow L^{p(x)}\left(\mathbb{R}^{m}\right)} .
$$

If we use Lemma 5, for $\epsilon>0$, then there exists a function $f \in C_{c}^{\infty}\left(\mathbb{R}^{m}\right)$, such that

$$
\frac{\left\|M_{a}^{c} f\right\|_{L^{p(x)}\left(\mathbb{R}^{m}\right)}}{\|f\|_{L^{p(x)}\left(\mathbb{R}^{m}\right)}}=\left\|M_{a}^{c}\right\|_{L^{p(x)}\left(\mathbb{R}^{m}\right) \rightarrow L^{p(x)}\left(\mathbb{R}^{m}\right)}-\epsilon .
$$

Since $f \in C_{c}^{\infty}\left(\mathbb{R}^{m}\right)$ implies $f \in L^{p(x)}\left(\mathbb{R}^{m}\right)$, we have $M_{a}^{c} f \in L^{p(x)}\left(\mathbb{R}^{m}\right)$. If $\mathbb{R}$ is a real integer, then we have

$$
\left\|\left(M_{a}^{c} f\right) \chi_{(|.| \geq \mathbb{R})}\right\|_{L^{p(x)}\left(\mathbb{R}^{m}\right)} \leq \epsilon\|f\|_{L^{p(x)}\left(\mathbb{R}^{m}\right)} .
$$

Now we set $\delta_{0}=\mathbb{Z}_{+}+\mathbb{R}$ ( $\mathbb{Z}_{+}$is a positive integer and $\mathbb{R}$ is a real integer). Then it can be written from the definition of $M_{a, \delta}^{c}$ that

$$
M_{a}^{c} f(x)=M_{a, \delta_{0}}^{c} f(x),
$$


holds for $|x|<\mathbb{R}$. Hence, from (20)-(22), we obtain

$$
\begin{gathered}
\left\|M_{a, \delta_{0}}^{c} f\right\|_{L^{p(x)}\left(\mathbb{R}^{m}\right)} \geq\left\|\left(M_{a, \delta_{0}}^{c} f\right) \chi_{(|x|<R)}\right\|_{L^{p(x)}\left(\mathbb{R}^{m}\right)} \\
=\left\|\left(M_{a}^{c} f\right) \chi_{(|x|<R)}\right\|_{L^{p(x)}\left(\mathbb{R}^{m}\right)} \\
\geq\left\|M_{a}^{c} f\right\|_{L^{p(x)}\left(\mathbb{R}^{m}\right)}-\left\|\left(M_{a}^{c} f\right) \chi_{(|x| \geq R)}\right\|_{L^{p(x)}\left(\mathbb{R}^{m}\right)} \\
\geq\left\|M_{a}^{c}\right\|_{L^{p(x)}\left(\mathbb{R}^{m}\right) \rightarrow L^{p(x)}\left(\mathbb{R}^{m}\right)}\|f\|_{L^{p(x)}\left(\mathbb{R}^{m}\right)}-2 \epsilon\|f\|_{L^{p(x)}\left(\mathbb{R}^{m}\right)} .
\end{gathered}
$$

Obviously, (23) implies that

$$
\frac{\left\|M_{a, \delta_{0}}^{c} f\right\|_{L^{p(x)}\left(\mathbb{R}^{m}\right)}}{\|f\|_{L^{p(x)}\left(\mathbb{R}^{m}\right)}} \geq\left\|M_{a}^{c}\right\|_{L^{p(x)}\left(\mathbb{R}^{m}\right) \rightarrow L^{p(x)}\left(\mathbb{R}^{m}\right)}-2 \epsilon .
$$

Here, the inequality (24) yields

$$
\left\|M_{a, \delta_{0}}^{c}\right\|_{L^{p(x)}\left(\mathbb{R}^{m}\right) \rightarrow L^{p(x)}\left(\mathbb{R}^{m}\right)} \geq\left\|M_{a}^{c}\right\|_{L^{p(x)}\left(\mathbb{R}^{m}\right) \rightarrow L^{p(x)}\left(\mathbb{R}^{m}\right)}-2 \epsilon .
$$

From (19) and (25), we have

$$
\left\|M_{a, \delta}^{c}\right\|_{L^{p(x)}\left(\mathbb{R}^{m}\right) \rightarrow L^{p(x)}\left(\mathbb{R}^{m}\right)}=\left\|M_{a}^{c}\right\|_{L^{p(x)}\left(\mathbb{R}^{m}\right) \rightarrow L^{p(x)}\left(\mathbb{R}^{m}\right)^{\prime}}
$$

for $\forall \delta>0$ and $1 \leq p(x)<\infty$. Thus, proof of Theorem 3 is complete.

Now, let us prove the weak $(1,1)$ boundedness for the restricted centered diamond- $\alpha$ fractional maximal operator.

Theorem 4. If $M_{a, \delta}^{c}$ is defined by (5) and $1 \leq p(x)<\infty$, then

$$
\left\|M_{a, \delta}^{c}\right\|_{L^{1}\left(\mathbb{R}^{m}\right) \rightarrow L^{1, \infty}\left(\mathbb{R}^{m}\right)}=\left\|M_{a}^{c}\right\|_{L^{1}\left(\mathbb{R}^{m}\right) \rightarrow L^{1, \infty}\left(\mathbb{R}^{m}\right)}
$$

holds for all $\delta>0$.

Proof. Let $M_{a, \delta}^{c}$ be defined by (5) for $0<\delta<\infty$. First, we prove that

$$
\left\|M_{a, \delta}^{c}\right\|_{L^{1}\left(\mathbb{R}^{m}\right) \rightarrow L^{1, \infty}\left(\mathbb{R}^{m}\right)}=\left\|M_{a, 1}^{c}\right\|_{L^{1}\left(\mathbb{R}^{m}\right) \rightarrow L^{1, \infty}\left(\mathbb{R}^{m}\right)} .
$$

From the identity (17), we get

$$
M_{a, \delta}^{c} f(\delta x)=M_{a, 1}^{c}\left(\tau_{\delta} f\right)(x) .
$$

For any $0<\delta$, we obtain from (26) that

$$
\left|\left\{x: M_{a, 1}^{c}\left(\tau_{\delta} f\right)>\mu\right\}\right|=\left|\left\{x: M_{a, \delta}^{c} f(\delta x)>\mu\right\}\right|=\left|\left\{\frac{x}{y}: M_{a, \delta}^{c} f(x)>\mu\right\}\right|=\delta^{-m}\left|\left\{\frac{x}{y}: M_{a, \delta}^{c} f(x)>\mu\right\}\right| .
$$

Thus (27) implies that

$$
\sup _{\mu>0} \mu\left|\left\{x: M_{a, 1}^{c}\left(\tau_{\delta} f\right)>\mu\right\}\right|=\delta^{-m} \sup _{\mu>0}\left|\left\{\frac{x}{y}: M_{a, \delta}^{c} f(x)>\mu\right\}\right| .
$$

If $\|f\|_{L^{1}\left(\mathbb{R}^{m}\right)} \neq 0$, then it follows from (28) that

$$
\begin{gathered}
\frac{\delta^{-m} \sup _{\mu>0} \mu\left|\left\{x: M_{a, \delta}^{c} f(x)>\mu\right\}\right|}{\|f\|_{L^{1}\left(\mathbb{R}^{m}\right)}}=\frac{\sup _{\mu>0} \mu\left|\left\{x: M_{a, 1}^{c}\left(\tau_{\delta} f\right)(x)>\mu\right\}\right|}{\|f\|_{L^{1}\left(\mathbb{R}^{m}\right)}} \\
=\frac{\delta^{-m} \sup \mu\left|\left\{x: M_{a, \delta}^{c} f(x)>\mu\right\}\right|}{\left\|\tau_{\delta} f\right\|_{L^{1}\left(\mathbb{R}^{m}\right)}} .
\end{gathered}
$$


Now taking the supremum over all $f \in L^{1}\left(\mathbb{R}^{m}\right)$ with $\|f\|_{L^{1}\left(\mathbb{R}^{m}\right)} \neq 0$ for the two sides of (29), we obtain

$$
\left\|M_{a, \delta}^{c}\right\|_{L^{1}\left(\mathbb{R}^{m}\right) \rightarrow L^{1, \infty}\left(\mathbb{R}^{m}\right)}=\left\|M_{a, 1}^{c}\right\|_{L^{1}\left(\mathbb{R}^{m}\right) \rightarrow L^{1, \infty}\left(\mathbb{R}^{m}\right)} .
$$

Next, we will use (30) to prove that

$$
\left\|M_{a, \delta}^{c}\right\|_{L^{1}\left(\mathbb{R}^{m}\right) \rightarrow L^{1, \infty}\left(\mathbb{R}^{m}\right)}=\left\|M_{a}^{c}\right\|_{L^{1}\left(\mathbb{R}^{m}\right) \rightarrow L^{1, \infty}\left(\mathbb{R}^{m}\right)},
$$

holds for $0<\delta$. Now, let us prove the correctness of the following equation.

$$
\sup _{\mu>0} \mu d_{M_{a}^{c} f}(\mu)=\limsup _{\delta \rightarrow \infty} \mu d_{M_{a, \delta}^{c} f}(\mu),
$$

holds for any $f \in L^{1}\left(\mathbb{R}^{m}\right)$ with $\|f\|_{L^{1}\left(\mathbb{R}^{m}\right)} \neq 0$. Clearly, the right side of (31) is not bigger than the left side, so it is enough to show opposite inequality. From Lemma 3, we have

$$
\sup _{\mu>0} \mu d_{M_{a}^{c} f}(\mu)=\sup _{\mu>0} \mu\left(\lim _{\delta \rightarrow \infty} d_{M_{a, \delta}^{c} f}(\mu)\right) .
$$

Let $A=\sup _{\mu>0} \mu d_{M_{a}^{c} f}(\mu)$. For $0<\epsilon$, there must be a $\mu_{0} \in \mathbb{R}^{+}$such that

$$
A \geq \mu_{0} d_{M_{a}^{c} f}\left(\mu_{0}\right) \geq A-\epsilon .
$$

We conclude that

$$
A-\epsilon \leq \mu_{0} d_{M_{a}^{c} f}\left(\mu_{0}\right)=\lim _{\delta \rightarrow \infty} d_{M_{a, \delta}^{c} f}(\mu) \leq \sup _{\mu>0} \mu\left(\lim _{\delta \rightarrow \infty} d_{M_{a, \delta}^{c} f}(\mu)\right) .
$$

This is equivalent to $A \leq \sup _{\mu>0} \mu\left(\lim _{\delta \rightarrow \infty} d_{M_{a, \delta}^{c} f}(\mu)\right)$.

Herewith, (31) holds. If we use Equation (31), we obtain that

$$
\begin{aligned}
& \left\|M_{a}^{c}\right\|_{L^{1}\left(\mathbb{R}^{m}\right) \rightarrow L^{1, \infty}\left(\mathbb{R}^{m}\right)}=\sup _{\|f\|_{L^{1}\left(\mathbb{R}^{m}\right)} \neq 0} \frac{\sup _{\mu>0} \mu d_{M_{a}^{c} f}(\mu)}{\|f\|_{L^{1}\left(\mathbb{R}^{m}\right)}} \\
& =\sup _{\|f\|_{L^{1}\left(\mathbb{R}^{m}\right)} \neq 0} \frac{\limsup _{\delta \rightarrow \infty} \mu d_{M_{a, \delta}^{c} f}(\mu)}{\|f\|_{L^{1}\left(\mathbb{R}^{m}\right)}} \\
& =\lim _{\delta \rightarrow \infty} \sup _{\|f\|_{L^{1}\left(\mathbb{R}^{m}\right)} \neq 0} \frac{\sup _{\mu>0} \mu d_{M_{a, \delta}^{c}} f(\mu)}{\|f\|_{L^{1}\left(\mathbb{R}^{m}\right)}} \\
& =\lim _{\delta \rightarrow \infty}\left\|M_{a, \delta}^{c}\right\|_{L^{1}\left(\mathbb{R}^{m}\right) \rightarrow L^{1, \infty}\left(\mathbb{R}^{m}\right)} .
\end{aligned}
$$

Thus, we get the result we want to achieve.

Remark 2. Let $M_{a}$ be the uncentered fractional maximal operator defined by (3). Define the iterated fractional maximal operator denoted by $M_{a}^{i+1}$ as follows:

$$
M_{a}^{i+1} g(y)=M_{a}\left(M_{a}^{i} g\right)(y)
$$

for $i=1,2,3, \ldots$ and $y \in \mathbb{R}^{m}$. Set $\left(M_{a \delta}^{1} g\right)(y)=\left(M_{a} g\right)(y)$.

Lemma 6. Assume that a sequence $\left\{d_{k}\right\}_{k=1}^{\infty}$ satisfies the following two conditions simultaneously: (a) $d_{1}=s \in(0.1)$, 
(b) for any $i \geq 1, d_{i+1}=(1-s) d_{i}+s$.

Then $\left\{d_{k}\right\}_{k=1}^{\infty}$ is strictly monotone increasing and we have

$$
\lim _{i \rightarrow \infty} d_{i}=1
$$

Proof. By the mathematical induction and the two conditions (a) and (b), we can easily obtain $0<d_{i}<1$ for each $i \in \mathbb{N}$. Furthermore, the condition (b) implies

$$
d_{i+1}-d_{i}=(1-s) d_{i}+s-d_{i}=s\left(1-d_{i}\right)>0 .
$$

This shows that $\left\{d_{k}\right\}_{k=1}^{\infty}$ is strictly monotone increasing. Since $\left\{d_{k}\right\}_{k=1}^{\infty}$ is monotone increasing and has the upper bound, the limit of $\left\{d_{k}\right\}_{k=1}^{\infty}$ exists, and we can easily get

$$
\lim _{i \rightarrow \infty} d_{i}=1
$$

By Lemma 6, we have the following corollary.

Corollary 1. For any $g \in L^{\infty}\left(\mathbb{R}^{m}\right)$, the equation

$$
\lim _{i \rightarrow \infty} M_{a}^{i} g(y)=\|g\|_{\infty}
$$

holds for $i=1,2,3, \ldots$ and $y \in \mathbb{R}^{m}$.

\section{Conclusions}

For more than a quarter century, the concept of time scales has taken an important place in the literature. Mathematicians and scientists working in other disciplines have demonstrated many applications of dynamic equations and integral inequalities; for example, transformations, inverse conversions, extensions, wave equations, heat transfer, optics, fluid dynamics, quantum calculus, economy, etc. The boundedness and compactness of the integral operators we know from harmonic analysis occupy an important place in the literature. Norms in variable exponential spaces are used to solve these problems. Previously, studies on the concept of the equivalence of norms in variable exponential spaces were conducted. In this way, we obtain the boundedness and compactness of integral operators that we do not have any information about. For more detailed information, we refer the reader to references.

In this study, we wanted to relate the norms of integral operators with time scales. In this article, we showed the equivalence of the norm variable exponent $L^{p(x)}$ of the restricted centered fractional maximal diamond- $\alpha$ integral $M_{a, \delta}^{c}$ with norm variable exponent $L^{p(x)}$ of centered fractional maximal diamond- $\alpha$ integral $M_{a}^{c}$ for all $0<\delta<\infty$ and $1 \leq p(x)<\infty$ on time scales. Hereby, we will be able to establish the boundedness and compactness conditions of fractional integral operators. In the future, we plan to carry these studies to variable exponent grand Lebesgue spaces, which is more general.

Author Contributions: The author has read and agreed to the published version of the manuscript.

Funding: This work received no external funding.

Institutional Review Board Statement: Not applicable.

Informed Consent Statement: Not applicable.

Acknowledgments: The author would like to thank the editor and the referees for his/her careful reading and valuable comments.

Conflicts of Interest: The author declares that there is no conflict of interest. 


\section{References}

1. Hilger, S. Ein Maßkettenkalkül mit Anwendung auf Zentrmsmannigfaltingkeiten. Ph.D. Thesis, University Würzburg, Wuerzburg, Germany, 1988.

2. Agarwal, R.P.; Bohner, M.; Peterson, A. Inequalities on time scales: A survey. Math. Inequal. Appl. 2001, 2001, 535-555. [CrossRef]

3. Akin-Bohner, E.; Bohner, M.; Akin, F. Pachpatte inequalities on time scales. J. Inequalities Pure Appl. Math. 2005, 6, 1-23.

4. Li, W.N. Nonlinear integral inequalities in two independent variables on time scales. Adv. Differ. Equ. 2011, 2011, 283926. [CrossRef]

5. Anastassiou, G.A. Principles of delta fractional calculus on time scales and inequalities. Math. Comput. Model. 2010, 52, 556-566. [CrossRef]

6. Wong, F.-H.; Yeh, C.-C.; Yu, S.-L.; Hong, C.-H. Young's inequality and related results on time scales. Appl. Math. Lett. 2005, 18, 983-988. [CrossRef]

7. Wong, F.-H.; Yeh, C.-C.; Lian, W.-C. An extension of Jensen's inequality on time scales. Adv. Dynam. Syst. Appl. 2006, 1, 113-120.

8. Kuang, J. Applied İnequalities; Shandong Science Press: Jinan, China, 2003.

9. Uçar, D.; Hatipoğlu, V.F.; Akincali, A. Fractional integral inequalities on time scales. Open J. Math. Sci. 2018, 2, 361-370. [CrossRef]

10. Özkan, U.M.; Sarikaya, M.Z.; Yildirim, H. Extensions of certain integral inequalities on time scales. Appl. Math. Lett. 2008, 2, 993-1000. [CrossRef]

11. Tian, J.-F.; Ha, M.-H. Extensions of Hölder-type inequalities on time scales and their applications. J. Nonlinear Sci. Appl. 2017, 10, 937-953. [CrossRef]

12. Kac, V.; Cheung, P. Quantum Calculus; Universitext Springer: New York, NY, USA, 2002.

13. Yang, W.-G. A functional generalization of diamond- $\alpha$ integral Hölder's inequality on time scales. Appl. Math. Lett. 2010, 23, 1208-1212. [CrossRef]

14. Spedding, V. Taming nature's numbers. New Sci. 2003, 179, 28-31.

15. Tisdell, C.C.; Zaidi, A. Basic qualitative and quantitative results for solutions to nonlinear dynamic equations on time scales with an application to economic modelling. Nonlinear Anal. 2008, 68, 3504-3524. [CrossRef]

16. Bohner, M.; Heim, J.; Liu, A. Qualitative analysis of Solow model on time scales. J. Concr. Appl. Math. 2015, $13,183-197$.

17. Brigo, D.; Mercurio, F. Discrete time vs continuous time stock-price dynamics and implications for option pricing. Financ. Stochast 2000, 4, 147-159. [CrossRef]

18. Seadawy, A.R.; Iqbal, M.; Lu, D. Nonlinear wave solutions of the Kudryashov-Sinelshchikov dynamical equation in mixtures liquid-gas bubbles under the consideration of heat transfer and viscosity. J. Taibah Univ. Sci. 2019, 13, 1060-1072. [CrossRef]

19. Bohner, M.; Agarwal, R.P. Basic calculus on time scales and some of its applications. Result. Der Math. 1999, 35, 3-22.

20. Bohner, M.; Peterson, A. Dynamic Equations on Time Scales, An Introduction with Applications; Birkhauser: Boston, MA, USA, 2001.

21. Tuna, A.; Kutukcu, S. Some integral inequalities on time scales. Appl. Math. Mech. (Engl. Ed.) 2008, 29, 23-28. [CrossRef]

22. D'Ovidio, M.; Loreti, P.; Momenzadeh, A.; Ahrabi, S.S. Determination of Order in Linear Fractional Differential Equations. Fract. Calc. Appl. Anal. 2018, 21, 937-948. [CrossRef]

23. Heris, J.M.; Aghdaei, M.F. Equivalent HPM with ADM and Convergence of the HPM to a Class of Nonlinear Integral Equations. J. Math. Ext. 2013, 7, 33-49.

24. Heris, J.M.; Bagheri, M. Exact Solutions for the Modified KdV and the Generalized KdV Equations via Exp-Function Method. J. Math. Ext. 2010, 4, 75-95.

25. Akin, L. On the Fractional Maximal Delta Integral Type Inequalities on Time Scales. Fractal Fract. 2020, 4, 26. [CrossRef]

26. Higgins, R. Asymptotic behavior of second-order nonlinear dynamic equations on time scales. Discret. Contin. Dyn. Syst. Ser. B 2010, 13, 609-622. [CrossRef]

27. Ozturk, O.; Higgins, R. Limit behaviors of nonoscillatory solutions of three-dimensional time scale systems. Turk. J. Math. 2018, 42, 2576-2587. [CrossRef]

28. Shi, Z.S.H.; Yan, D.Y. Criterion on $\mathrm{L}^{\mathrm{P}_{1}} \times \mathrm{L}^{\mathrm{P}_{2}} \rightarrow \mathrm{L}^{\mathrm{q}}$-boundedness for oscillatory bilinear Hilbert transform. Abstr. Appl. Anal. 2014, 2014, 712051. [CrossRef]

29. Mingquan, W.; Xudong, N.; Di, W.; Dunyan, Y. A note on Hardy-Littlewood maximal operators. J. Inequalities Appl. 2016, 2016, 1-13.

30. Grafakos, L. Classical and Modern Fourier Analysis; China Machine Press: Beijing, China, 2005.

31. Rudin, W. Real and Complex Analysis, 3rd ed.; McGraw-Hill: Singapore, 1987.

32. Mamedov, F.I.; Zeren, Y.; Akin, L. Compactification of weighted Hardy operator in variable exponent Lebesgue spaces. Asian J. Math. Comput. Sci. 2017, 17, 38-47.

33. Akin, L. A Characterization of Approximation of Hardy Operators in VLS. Celal Bayar Univ. J. Sci. 2018, 14, 333-336. [CrossRef]

34. Akin, L.; Zeren, Y. Some properties for higher order commutators of Hardy-type integral operator on Herz-Morrey spaces with variable exponent. Sigma J. Eng. Nat. Sci. 2019, 10, 157-163.

35. Capone, C.; David Cruz-Uribe, S.F.O.; Fiorenza, A. The fractional maximal operator and fractional integrals on variable $L^{p}$ spaces. Rev. Mat. Iberoam. 2007, 23, 743-770. [CrossRef]

36. Zhang, P.; Wu, J. Commutators of the fractional maximal function on variable exponent Lebesgue spaces. Czechoslov. Math. J. 2014, 64, 183-197. [CrossRef] 
37. Beltran, D.; Madrid, J. Regularity of the centered fractional maximal Function on radial functions. J. Funct. Anal. 2020, 108686. [CrossRef]

38. Akin, L.; Dusunceli, F. A New Approach for Weighted Hardy's Operator in VELS. Appl. Math. Nonlinear Sci. 2019, 4, 417-432. [CrossRef]

39. Agarwal, R.P.; Otero-Espinar, V.; Perera, K.; Vivero, D.R. Basic properties of Sobolev's spaces on time scales. Adv. Differ. Equ. 2006, 2006, 38121.

40. Akin, L.; Zeren, Y. An investigation on fractional maximal operator in time scales. In Proceedings of the 3rd International E-Conference On Mathematical Advances And Applications, Yildiz Technical University, Istanbul, Turkey, $24-27$ June 2020.

41. Lu, S.Z.; Yan, D.Y. LP Boundedness of multilinear oscillatory singular integrals with Calderón-Zygmund kernel. Sci. China Ser. A 2002, 45, 196-213.

42. Agarwal, R.P.; O’Regan, D.; Saker, S.H. Hardy Type Inequalities on Time Scales; Springer: Cham, Switzerland, 2016.

43. Akin, L. On some results of weighted Hölder type inequality on time scales. Middle East J. Sci. 2020, 6, 15-22. [CrossRef] 